\title{
NUMERICAL STUDY OF CENTRAL NERVOUS SYSTEM, MUSCULO-SKELETAL SYSTEM, AND THEIR COUPLING TOWARD MOTOR DYSFUNCTION IN PARKINSON'S DISEASE
}

\author{
K. Shimizu ${ }^{1}$, N. Yamamura ${ }^{2}$, S. Takagi ${ }^{1}$ \\ ${ }^{1}$ Department of Mechanical Engineering, the University of Tokyo (kshimizu@fel.t.u- \\ tokyo.ac.jp)
}

${ }^{2}$ Computational Science Research Program, RIKEN

\begin{abstract}
In this research activity, we are now developing the numerical model for the motor dysfunction, for example Parkinson's disease, on a high performance computer. The final target of the activity is to develop the integration model consisting of neural networks (brain, central nervous system, and so on) and three-dimensional musculo-skeletal system, and to reproduce primary symptoms of Parkinson's disease in order to clarify the cause of the motor dysfunction and obtain the knowledge about effective treatments. The developed central nervous system includes major motoneurons related to a motion (e.g. $\alpha$-motoneuron) and described as simultaneous ordinary differential equations, in which communications of excitatory and/or inhibitory signals with each other are expressed mathematically The musculoskeletal model is based on a nonlinear finite element framework for incompressible hyperelastic materials, and can treat both passive behavior of biological soft tissues and active behavior of muscle fibers. The model can successfully reproduce the muscle-tendon behavior and generated force during an isometric contraction.
\end{abstract}

Keywords: Parkinson's disease, central nervous system, musculo-skeletal system

\section{INTRODUCTION}

Parkinson's disease is a degenerative disorder of central nervous system and causes motor dysfunction. The motor dysfunction is generally considered to be caused by an abnormal motor command from the basal ganglia, which results from the death of dopamineproducing cells in a midbrain region. However, the cause of the depletion is not clarified yet. Therefore, treatments remain palliative and its development cannot be stopped.

In this research, we are now developing a numerical model for the motor dysfunction in Parkinson's patients on a high performance computer system. The final target of this study is to develop the integrated model of brain, central nervous system, and three-dimensional musculo-skeletal system, in order to clarify the cause of the motor dysfunction in Parkinson's patients and obtain the knowledge about effective treatments. 
The final objective of this research, therefore, is to develop a numerical model consisting of a brain, neural networks, and musculo-skeletal system for a human motion and to reproduce primary symptoms of Parkinson's disease on a high performance computer, in order to clarify the cause of the motor dysfunction and obtain the knowledge about effective treatments. In the current status, central nervous model and skeletal muscle model are developed separately, then these models are integrated.

\section{NEURAL NETWORKS}

To reproduce a motion of a human in a numerical simulation, development of the models for brain, neural networks, and musculo-skeletal system and integration of their models are required. Numerical results by Cutsuridis [1] are given as a successful work about numerical simulation of Parkinson's disease. In his model, activities of primary motoneurons and interneurons related to an arm motion are expressed as simultaneous ordinary differential equations. His results show dopamine depletion in a brain causes abnormal muscle behaviors and neuron activities which are similar to rigidity in Parkinson's patients. Meanwhile, many researchers are now developing numerical models for the brain (consisting of globus pallidus externa, globus pallidus interna, subthalamic nucleus, and so on) of Parkinson's patients and investigating the influences of dopamine depletion generating an abnormal motor command. In such research activities, membrane potentials of neurons are expressed as ordinary differential equations and the voltage depends on the behavior of ion channels. As mentioned above, our final target is to develop the integration model, and the central nervous system plays a role in transmitting signals from brain to muscle. Accordingly, an analogous strategy to a brain model is adopted for the neural networks for a motion in view of the connection of brain and muscle behavior.

To realize this concept, we follow the success of the numerical model developed by Cisi et al. [2], which simulation system consisting of the spinal cord circuitry and associated muscles can successfully reproduce force generation and motonueron recruitment. Mathematical treatments for neurons forming neural networks for motor command communication are based on a compartment model and expressed as ordinary differential equations for membrane potential. As an example, the expressions for an $\alpha$-motoneuron are given below:

$$
\begin{aligned}
& C_{s} \frac{d V_{s}}{d t}=-I_{s y n, s}-g_{l s}\left(V_{s}-E_{l}\right)-g_{c}\left(V_{s}-V_{d}\right)-I_{\text {ion }}, \\
& C_{d} \frac{d V_{d}}{d t}=-I_{s y n, d}-g_{l d}\left(V_{d}-E_{l}\right)-g_{c}\left(V_{d}-V_{s}\right), \\
& I_{\text {ion }}=g_{N a} m^{3} h\left(V_{s}-E_{N a}\right)+g_{K f} n^{4}\left(V_{s}-E_{K f}\right)+g_{K s} q^{2}\left(V_{s}-E_{K s}\right), \\
& C_{s}=2 \pi r_{s} l_{s} C_{m}, \\
& C_{d}=2 \pi r_{d} l_{d} C_{m} .
\end{aligned}
$$


Here, $V_{s}$ is a membrane potential for soma, $V_{d}$ is a membrane potential for dendrite (two compartment model is employed for $\alpha$-motoneuron), $m, h, n$, and $q$ are gate variables for corresponding ion channels. The three kinds of ion channels (sodium, fast potassium, and slow potassium) are considered in the model. The size principle can be reproduced by means of changing the size (radius : $r_{s}$, length $: l_{s}$, and so on) of each neuron. An example of the soma diameter is shown in Fig. 1. In this figure, 800 S-type motoneurons, 50 FR-type motoneurons, and 50 FF-type motoneurons are illustrated, and they have linear slope in diameter.

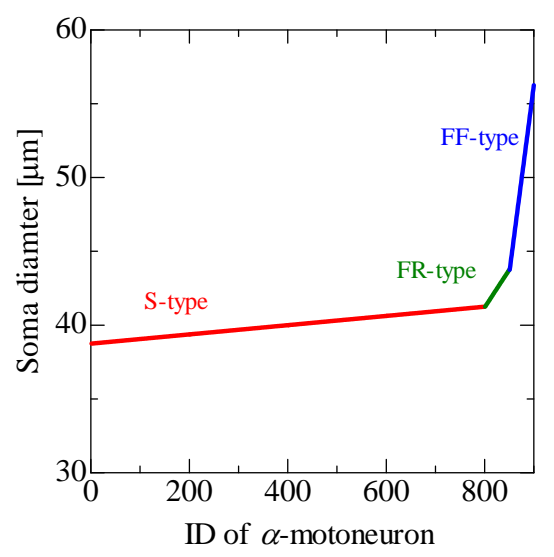

Figure 1. Soma diameter for three types of $\alpha$-motoneurons.

Behaviors of motoneurons obtained by numerical tests for the above model are illustrated in Fig. 2, in which direct currents of 5, 10, and $50 \mathrm{nA}$ are injected. It is shown from this figure that small direct current can cause firing of only small $\alpha$-motoneuron. When the injected current becomes larger, larger $\alpha$-motoneuron can be activated. In this test, all of the $\alpha$ motoneurons are activated for the injected current of $50 \mathrm{nA}$. This result shows the size principle is successfully reproduced by this numerical modeling for $\alpha$-motoneuron. Figure 3 shows generated force when the direct current is injected. The generated force becomes large gradually when the injected current becomes large. The maximum force is realized at the injected current of $50 \mathrm{nA}$. The increase in the injected current can't change the generated force when the current is larger than $50 \mathrm{nA}$. This corresponds to the behavior of motoneurons shown in Fig. 3, which indicates successful reproduction of size principle of motoneurons. 

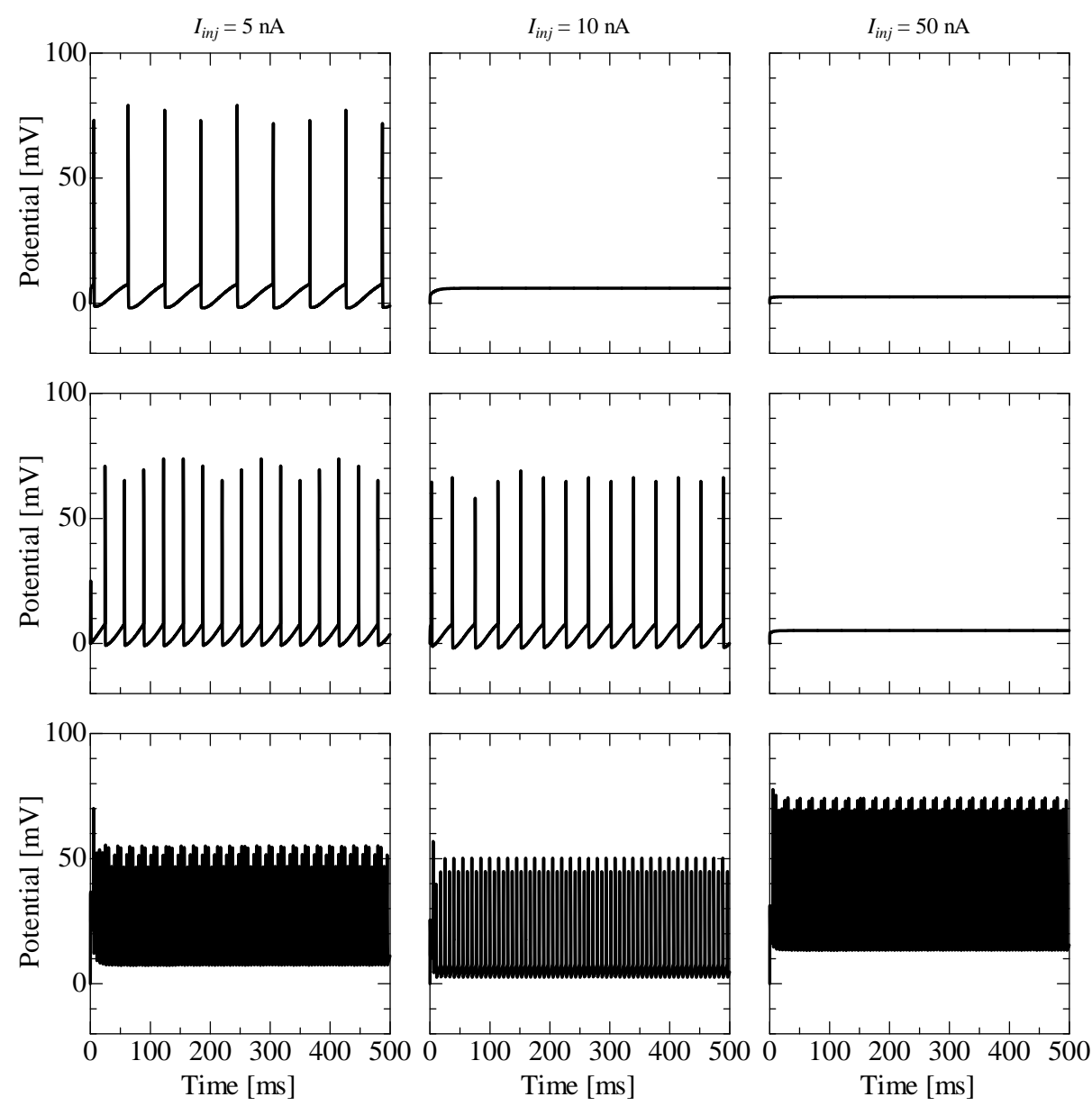

Figure 2. Time train of membrane potential of $\alpha$-motoneurons for three kinds of injected currents. The column means injected direct currents: 5, 10, and $50 \mathrm{nA}$ from left to right. The row shows the membrane potential of each $\alpha$-motoneuron: the smallest S-type neuron, the largest FR-type neuron, and the largest FF-type neuron from the top to the bottom.

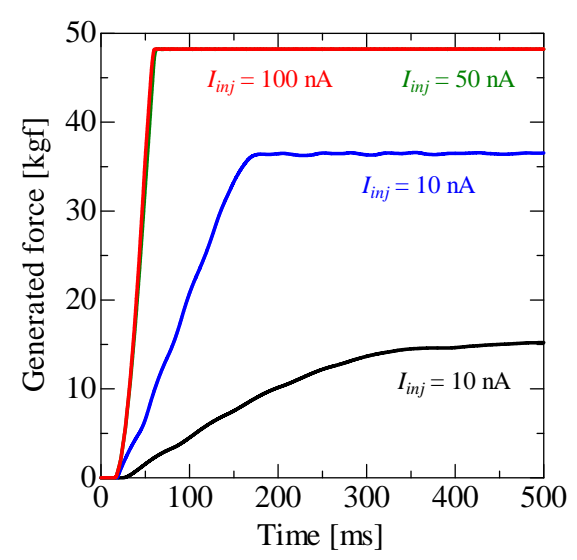

Figure 3. Generated force against injected direct current. 


\section{MUSCULO-SKELETAL SYSTEM}

The numerical modeling for musculo-skeletal system is based on a nonlinear finite element method under the assumption of incompressibility of biological soft tissues such as muscle and tendon. Here, a mixed type displacement-pressure finite element formulation, a total Lagrangian formulation, and a fully implicit time integration procedure are adopted. One of the characteristics of the biological tissues is its ability to develop internal forces, therefore, the stress state in a muscle must be expressed as the result of a superposition of passive and active parts, i.e. [4]

$$
\sigma_{f}^{\text {total }}=\sigma_{f}^{\text {pas }}+\sigma_{f}^{a c t}
$$

where $\sigma_{f}^{\text {total }}, \sigma_{f}^{\text {pas }}$, and $\sigma_{f}^{\text {act }}$ are the total, the passive, and the active Cauchy stress normal components in the muscle fiber direction.

For the biological soft tissues, the strain energy density function can be written as [5]

$$
W=\bar{W}+W^{v o l}+Q
$$

The term $\bar{W}$ is the contribution of the isochoric strain energy and is a function of the adopted hyperelastic constitutive model. The second term $W^{\text {vol }}$ represents the strain energy associated with the volumetric change, and the third term $Q$, which comes from the introduction of incompressibility of the biological soft tissues, is the function of both displacement and separately interpolated pressure. The implemented model for the passive part of the muscle is assumed to be the anisotropic material ones, i.e: [4]

$$
\bar{W}^{\text {muscle }}=\bar{W}^{\text {matrix }}+\bar{W}^{\text {fiber }} \text {. }
$$

The first term $\bar{W}^{\text {matrix }}$ corresponds to the connective tissues of muscles and is defined as a Mooney-Rivlin material:

$$
\bar{W}^{\text {matrix }}=\sum_{i+j=1}^{2} c_{i j}\left(\bar{I}_{1}-3\right)^{i}\left(\bar{I}_{2}-3\right)^{j}
$$

where $c_{i j}$ are material constants, $\bar{I}_{1}$ and $\bar{I}_{2}$ are reduced invariants right Cauchy-Green strain tensor, respectively. The second term $\bar{W}^{\text {fiber }}$ represents the muscle fibers and connects the Cauchy stress in the fiber direction $\left(\sigma_{\text {fiber }}^{\text {pas }}\right)$ to the fiber stretch $(\lambda)$ by the relation

$$
\lambda \frac{\partial \bar{W}^{\text {fiber }}}{\partial \lambda}=\sigma_{\text {fiber }}^{\text {pas }}=\sigma_{0}^{\text {pas }} \frac{\lambda}{\lambda_{0}} f_{\text {fiber }}^{\text {pas }},
$$

where $\sigma_{0}^{\text {pas }}$ is a material constant and $f_{\text {fiber }}^{\text {pas }}$ is the normalized passive force given by

$$
f_{\text {fiber }}^{\text {pas }}\left\{\begin{array}{cc}
0 & \text { for } \lambda^{*} \leq 1 \\
\gamma_{1}\left[\exp \left\{\gamma_{2}\left(\lambda^{*}-1\right)\right\}-1\right] & \text { for } 1<\lambda^{*} \leq 1.4 \\
\gamma_{1} \gamma_{2} \exp \left(0.4 \gamma_{2}\right)\left(\lambda^{*}-1.4\right)+\gamma_{1}\left[\exp \left(0.4 \gamma_{2}\right)-1\right] & \text { for } \lambda^{*}>1.4
\end{array}\right.
$$


where $\lambda^{*}$ is the normalized stretch defined as $\lambda^{*}=1 / \lambda_{0}$. The models for tendon is assumed to be isotropic and expressed as Mooney-Rivlin model.

The three-dimensional Hill-type model [4], which is written as Eq. (12), is used for the muscle active behavior.

$$
\sigma_{\text {fiber }}^{a c t}=\sigma_{0}^{i s o m} \frac{\lambda}{\lambda_{0}} \cdot f_{l} \cdot f_{v} \cdot f_{t}
$$

In the above expression, $\quad \sigma_{0}^{\text {isom }}$ is the maximum isometric stress occurring at the optimum fiber stretch $\lambda_{0}$. $f_{l}, f_{v}$, and $f_{t}$ are the activation function, length-dependence function, and velocity-dependence function, respectively.

Numerical results for isometric contraction of a human triceps surae muscle are shown in Fig. 4. In this calculation, modeled surae muscle is based on medical images. It is seen that each muscle causes a contraction in the fiber directions. The Achilles tendon is stretched by the aponeurosis existing between gastrocnemius and soleus, resulting in the force generation.
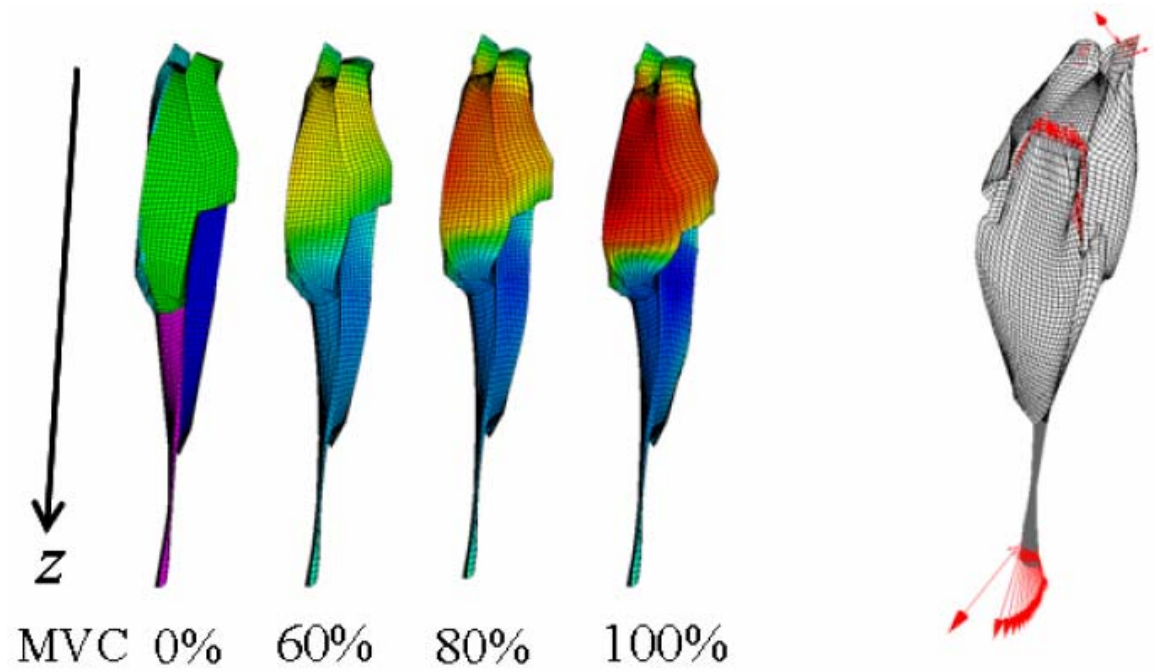

Figure 4. Simulation results of the isometric contraction of a human triceps surae muscle. Four figures in the left-hand side illustrate the deformation and displacement of the longitudinal direction. The right-hand side figure shows the nodal force vectors at the end of the contraction.

The effects of the maximum isometric stress on the generated force at the insertion of Achilles tendon at the end of the activation are also examined. Figure 5 shows the numerical results of generated force against maximum isometric stress which is varied from 0.05 to 1.0 MPa based on the literature. The maximum isometric stress increases the generated force. The largest force, $\sigma_{0}^{\text {isom }}=1.0 \mathrm{MPa}$, is about 9.7 times larger than the smallest one, $\sigma_{0}^{\text {isom }}=0.05$ MPa. 


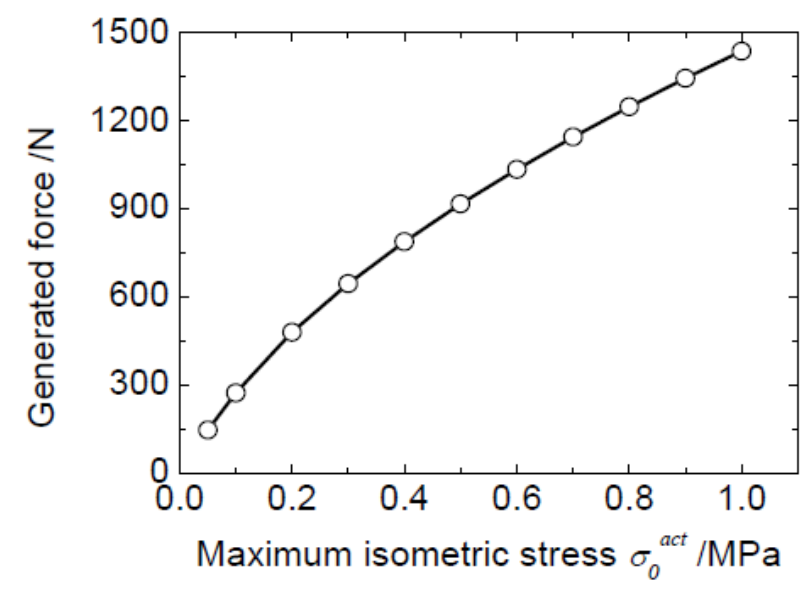

Figure 5. Generated force against the maximum isometric stress at the insertion of Achilles tendon at the end of the activation.

\section{SUMMARY}

In this paper, numerical models for the central nervous system and three-dimensional musculo-skeletal fininte element model are described. It is confirmed that the threedimensional mechanism of the force generation at Achilles tendon is obtained from the present musculo-skeletal model based on a finite element approach. And the numerical model for motoneurons reproduces recruitment during force generation. The final target of our research activity is to integrate these models together and to reproduce a human motion, primary symptoms of Parkinson's patients on a high performance computer. In the future, therefore, we are going to integrate these models, and furthermore brain model.

\section{REFERENCES}

[1] Vassilis Cutsuridis, "Origins of a repetitive and co-contractive biphasic pattern of muscle activation in Parkinson’s disease”, Neural Networks, 24, 592-601, 2011.

[2] Steven J. Schiff, “Towards model-based control of Parkinson's disease”, Phil. Trans. $R$. Soc. A, 368, 2269-2308, 2010.

[3] Rogerio R. L. Cisi, Andre F. Kohn, "Simulation system of spinal conrd motor nuclei and associated berves ans muscles, in a Web-based architecture”, J. Comput. Neurosci., 25, 520-542, 2008.

[4] Johansson, T., Meier, P., Blickhan, R., “A Finite-Element Model for the Mechanical Analysis of Skeletal Muscles”, J. Theor. Biol., 206, 131-149, 2000.

[5] Sussman, T., Bathe, K.-J., “A FE Formulation for Nonlinear Incompressible Elastic and Inelastic Analysis”, Computer \& Structures, 26, 357-409, 1987. 